\title{
Cost-effectiveness of population-based screening for colorectal cancer
}

\author{
J F O’Mahony*,1 \\ ${ }^{1}$ Department of Health Policy and Management, Trinity College Dublin, 2-4 Foster Place, Dublin 2, Ireland
}

Sir,

Sharp et al's (2012) cost-effectiveness analysis (CEA) of colorectal screening in Ireland sees the academic publication of a CEA published by Ireland's Health Information and Quality Authority (HIQA, 2009). Both publications deserve comment regarding the choice of alternatives compared and the confusion of average and incremental cost-effectiveness ratios (ACERs and ICERs, respectively).

First, regarding the number of alternatives compared. Both analyses describe a core of three screening strategies, biennial guaiac-based fecal occult blood testing between ages 55 and 74, biennial fecal immunochemical testing (FIT) between 55 and 74 and flexible sigmoidoscopy at 60 , and five additional strategies with alternative age ranges. No screening frequency other than biennial is considered. No start age below 55 or stop age above 74 is considered. No FIT test quantitative cutoff level other than of $100 \mathrm{ng} \mathrm{ml}^{-1}$ haemoglobin is considered. The primary cost-effectiveness results reported in Sharp et al's abstract are for the three core strategies.

The total of eight screening strategies is a relatively narrow set of alternatives. The core of three is even narrower. No rationale is given for the consideration of three core strategies or their significance relative to the others considered. The choice of a narrow range of alternatives is potentially problematic, as it can result in the omission of other relevant alternatives that may be more cost-effective. Furthermore, the omission of relevant alternatives can bias the ICERs of the included strategies downwards.

The narrow set of alternatives also has implications for uncertainty analysis. Sharp et al provide some summary estimates of cost-effectiveness acceptability curves. They report that FIT between 55 and 74 would have a $99 \%$ probability of being cost-effective at a threshold of $€ 14000$ /quality adjusted life year (QALY). However, this probability is not very meaningful, as no strategy more intensive than FIT between 55 and 74 is analysed: the apparent confidence in cost-effectiveness is due to the omission of relevant alternatives rather than a high degree of certainty.

Sharp et al cite Wilschut et al's (2011) analysis of alternative FIT cutoff levels, which found all strategies with a cutoff of $50 \mathrm{ng} \mathrm{ml}^{-1}$ to dominate all others. Sharp et al state that screening test sensitivity is not a major driver of cost-effectiveness and that their conclusions would not change if they repeated their analysis in light of this recent evidence. This is doubtful. If on repeating the analysis Sharp et al also found that a lower FIT cutoff dominates in all strategies, then they would not conclude that FIT screening with a $100-\mathrm{ng} \mathrm{ml}^{-1}$ cutoff is cost-effective. Wilschut et al's results suggest that Ireland's population colorectal screening programme will be unnecessarily inefficient unless the cutoff level is adjusted downwards from the $100 \mathrm{ng} \mathrm{ml}^{-1}$ assessed by Sharp et al.

Second, regarding the confusion between ACERs and ICERs. The cost-effectiveness ratios reported in Table 2 in Sharp et al and the primary results reported in the abstract are described as ICERs, but should be described as ACERs, as they are the ratio of costs and effects of each alternative relative to no screening. The received interpretation of ICERs is given by Siegel et al (1996b), whereby ICERs should be estimated as the incremental cost-effectiveness only between those strategies not subject to dominance or extended dominance, that is, those on the efficient frontier. ICERs not ACERs are the appropriate metric for determining cost-effectiveness (Siegel et al, 1996a).

The final paragraph of the results section does correctly report the ICERs for the efficient alternatives, but these are not the ICERs reported in the abstract or Table 2. This distinction matters. The ACER of FIT between 55 and 74 erroneously reported in the abstract and Table 2 as an ICER is approximately $€ 1,700 /$ QALY, whereas the correct ICER relative to FIT between 55 and 64 is $€ 3,200 / Q A L Y$ : the difference is almost a factor of two. Furthermore, reporting an ICER for gFOBT implies it is efficient, however it is not, as it is dominated by other strategies.

The consequence of this confusion in Sharp et al and HIQA's CEA is an abundance of irrelevant cost-effectiveness ratios being reported as ICERs. Both publications only needed to report three ICERs; one each for the three efficient strategies. Reporting superfluous cost-effectiveness ratios as ICERs can only confuse decision makers, especially when multiple ICERs are reported for the same strategy and ICERs are reported for inefficient strategies. This confusion of ICERs and ACERs has been further 
compounded by HIQA, as the 2009 CEA is used as an illustrative example of the reporting of cost-effectiveness estimates in their methods guidance (HIQA, 2010).

The number of alternatives considered within CEAs will be bound by data and other constraints. However, unnecessary constraints on the number of alternatives considered should not be imposed, such as the choice of three core strategies in this case. Analysts should provide decision makers with the correct interpretation of their costeffectiveness estimates and avoid unnecessary confusion.

\section{REFERENCES}

HIQA (2009) Health Technology Assessment of A Population-Based Colorectal Cancer Screening Programme in Ireland. Health Information and Quality Authority: Dublin.
HIQA (2010) Guidelines for the Economic Evaluation of Health Technologies in Ireland. Health Information and Quality Authority: Dublin.

Sharp L, Tilson L, Whyte S, O'Ceilleachair A, Walsh C, Usher C, Tappenden P, Chilcott J, Staines A, Barry M, Comber H (2012) Cost-effectiveness of population-based screening for colorectal cancer: a comparison of guaiacbased faecal occult blood testing, faecal immunochemical testing and flexible sigmoidoscopy. Br J Cancer 106: 805-816.

Siegel JE, Weinstein MC, Russell LB, Gold MR (1996a) Recommendations for reporting cost-effectiveness analyses. JAMA 276: 1339-1341.

Siegel JE, Weinstein MC, Torrance GW (1996b) Reporting cost-effectiveness studies and results. In Cost-Effectiveness in Health and Medicine, Gold MR, Siegel JE, Russell LB, Weinstein MC (eds), pp 276-303. Oxford University Press: New York.

Wilschut JA, Hol L, Dekker E, Jansen JB, van Leerdam ME, Lansdorp-Vogelaar I, Kuipers EJ, Habbema JDF, van Ballegooijen M (2011) Cost-effectiveness analysis of a quantitative immunochemical test for colorectal cancer screening. Gastroenterology 141: 1648-1655, e1. 